\title{
La Educación Ambiental no Formal orientada en la Pedagogía de la Esperanza
}

\section{Non-Formal Environmental Education ori- ented in the Pedagogy of Hope}

Luis Daniel Germer*

germer.daniel@gmail.com

\section{Resumen}

A mediados del año 2017, cercanos a terminar la segunda década del siglo XXI, la humanidad se debate ante el terror de un planeta sumido en el cambio climático, la extinción de las especies y pérdida de los bosques. Estas condiciones generan controversia, temor y en consecuencia desánimo en la población a nivel mundial. En el caso específico de Honduras se han dado inclusiones considerables de la temática ambiental en la educación aumentando así la conciencia de las personas. En este estudio se comenta la aplicación de la esperanza en el ámbito no formal en aras de impulsar un pensamiento autocrítico que permita al ciudadano integrarse en un mundo cambiante en donde es parte, y se convierte como tal, en voz activa del cambio que desea en su hogar, comunidad y planeta. El trabajo del educador para crear esos espacios de autorreflexión y generar así esperanza es crucial para comenzar un cambio. Por ello se sugiere en este trabajo el uso de los objetivos de desarrollo del milenio de las Naciones Unidas ya que plantean elementos de prosperidad y bienestar intrínsecos a toda la humanidad.

* Licenciado en Biología, Investigador independiente de Hondubirding Roots. Recibido 27 de agosto 2017 / Aceptado 18 de octubre 2017. 
Palabras clave: autocrítica, pesimismo, recursos naturales, objetivos de desarrollo del Milenio.

\begin{abstract}
In the middle of 2017, near the end of the second decade of the twenty-first century, humanity is struggling with the terror of a planet beset by climate change, extinction of species and loss of forests. These instances generate controversy, fear and consequently discouragement in the world population. There have been considerable inclusions of environmental issues in Honduran education raising people's awareness. This time, emphasis will be placed on the application of hope in non-Formal education in rela-

that allows the citizen to integrate itself in a changing world where he is part and must become as such an active voice of the change he wants in his home, community and planet.

The work of the educator becomes crucial to create these spaces of self-reflection in the process of sowing hope to begin a change. The text suggests using the United Nations Millennium Development Goals as they touch upon elements of prosperity and well-being intrinsic to all of humanity.
\end{abstract} tion to boost a self-critical thought

Keywords: self-criticism, pessimism, natural resources, Millennium Development Goals.

\title{
Introducción
}

La educación ambiental se ha convertido en un proyecto cada vez más relevante en las aulas de clase y en las capacitaciones sobre temáticas "ambientalistas y conservacionistas" en el ámbito no formal. A partir de la propia experiencia en el campo profesional, se aprecia que las 
personas comienzan a entender la problemática planetaria y las consecuencias de la degradación ambiental. Sin embargo, no logran dar el paso hacia la concienciación y se muestran imbuidos en la crítica del sistema actual, el resentimiento hacia el desarrollo y una apatía por cambiar sus estilos de vida, insistiendo en el conformismo asentado en los medios de vida consumistas en donde "tener más" es el equivalente de "ser más".

La educación ambiental ha fallado en inspirar hacia un mundo mejor, dejando únicamente la enseñanza de la culpa del hombre ante la degradación de los recursos naturales. A través de este escrito se espera lograr un espacio de autorreflexión para los educadores y de esta manera construir ideas para una educación ambiental efectiva que informe y genere opciones para el cambio brindando así esperanza en las personas.

\section{Métodos y materiales}

El presente documento surge como una asignación monográfica de “Corrientes Contemporáneas en la Educación", módulo de estudio dentro de la Maestría en Educación de las Ciencias Naturales de la Universidad Pedagógica Nacional Francisco Morazán, en su octava promoción. Se tomó como punto de partida la experiencia del autor en la educación no formal ambiental y la investigación acerca de los recursos naturales en Honduras. A partir de esta experiencia y crítica empírica de la situación se consultó una variedad de referencias bibliográficas sobre la educación no formal y los objetivos de la educación ambiental. Con la multitud de ideas enlazadas, se construyó una contrapropuesta analítica que conecta las principales ideas del tema

El escrito apunta a establecer ideas para abordar la educación ambiental a nivel no formal con un enfoque y necesidad cultural específica que informe, critique y construya a través de la esperanza, un cambio actitudinal en la población expuesta a la enseñanza. La 
educación ambiental tiene como función crear la conciencia en el humano sobre su entorno y sensibilizarle para que incida en los cambios para un mundo mejor. Se considera que la educación ambiental se ha estancado en repetir y realzar la situación actual de degradación de la naturaleza y recursos naturales sin presentar opciones concretas para un desarrollo sostenible (Fernández Bregón, 2011). Esta realidad afecta la sensibilización y genera desesperanza cimentando el consumismo desmedido ante el precepto de "Si ya viene el fin, ¿Por qué cambiar?".

\section{Discusión teórica}

\section{La esperanza como un modelo a implementar}

Freire (1996) en Pedagogía de la Esperanza sugiere que "la esperanza es una necesidad ontológica". Siendo así, ¿Cómo brindar esa esperanza y quienes la necesitan? La esperanza no es algo que se puede dar como algo tangible. La esperanza está en las interacciones de las personas que se desenvuelven en sociedad, o en este escenario, las que el educador no formal desarrolla con su audiencia.

El cambio como un estilo de vida para mejorar, es precedido por un proceso de crítica, pero sin que se convierta en un círculo vicioso. Existe un llamado al cambio social a partir de la crítica de la situación actual de las cosas, y aun hoy pareciera que la crítica domina apabullante sin el establecimiento de una nueva visión de las cosas. Para que exista esperanza es necesaria la crítica. La esperanza no puede existir sin desesperanza, existe una relación entre ellas, a medida una pierde territorio, la otra toma campo (Reyes García, 1995). De ahí que procesos permanentes de crítica sin una sugerencia o propuesta de cambio generan mayor desesperanza. Por tanto se vuelve fundamental equilibrar el discurso crítico y añadirle un impulso de acción hacia adelante.

Para implementar la esperanza, es necesario enfocar las necesidades y aspiraciones de las personas y sus comunidades locales (persona- 
familia-barrio-población) y evitar visiones nacionales insertadas como política particular de gobierno. Se ha observado que durante una capacitación no formal, la audiencia podrá escuchar con atención la retórica detrás del reciclaje, pero muy poco interés tendrán en aplicarlo, si este no incide directamente sobre sus medios de vida y propósitos. Para que la esperanza sea real, debe asirse de las experiencias vividas de las personas, y no afianzada en políticas particulares académicas o como parte de una agenda corporativa externa. A partir únicamente de la explicación magistral y la crítica, es difícil inspirar en las personas la actuación, en particular cuando el educador resulta ser una persona foránea de la comunidad.

A medida que se intenta dar esperanza lo que se produce es pesimismo. Este es el caso particular de la educación ambiental, la cual se ha enfocado en brindar conceptos abstractos y ha convertido la enseñanza en un manual de "yo pecador" de la conservación de los recursos naturales. Esta sensación de culpa genera pesimismo y oscurece la solución del problema. La educación ambiental ha informado sí, pero ha comunicado un mensaje de tristeza y fallado en dar esperanza. Quizá esto ha sido producto de la propia desesperanza de los tomadores de decisiones o un proyecto sistemático de generar temor a la humanidad para mantenerla en un estado de sumisión socioeconómico (Pain, 2009). Al respecto LeGrange (2011) comenta que la esperanza se presta como herramienta política populista debido a que presenta una extensa variedad de temas como la enfermedad, la pobreza, la ecología y el crimen, entre otros. Es decir, que se puede hablar de esperanza quitando esperanza a la humanidad. Siendo este el escenario, el tema del cambio climático se presenta ideal para generar temor en las personas (La Prensa, 2017).

Van Heertum (2006) sugiere que la crítica del orden actual de las cosas es necesaria para cualquier proyecto de cambio. Sin el análisis de la mala actuación, se hace complicado convencer de la necesidad de un cambio. Esta es la dificultad que se presenta en el escenario de la educación ambiental no formal para adultos, entonces ¿Cómo lograr convencer a las personas de hacer en su vida diaria un uso ético y 
responsable los recursos naturales? Es necesario encontrar algo que se desea lograr y a la vez algo que se quiera eliminar, es decir, cambiar desesperanza por esperanza. La esperanza se convierte en el complemento a la crítica. Se avanza hacia un discurso de acción que capitalice en los deseos reprimidos y brinde una alternativa provisional que motive a las personas a realizarlo (Freire, 1996).

La Educación Ambiental de la Esperanza y los Objetivos de Desarrollo del Milenio

Novo (1995) plantea que la educación ambiental es un pronunciamiento hacia la ética planetaria, sugiere la idea de responsabilidad, equilibrio y decencia hacia el vecino; una educación que se aleja de la mirada antropocéntrica de la educación y se inserta en una visión ecocéntrica. La educación ambiental aboga por una humanidad en armonía con la naturaleza como una posibilidad necesaria y desafiante ante la cual el planeta, la sociedad y la vida son espacios de posibilidades.

Nieto-Caraveo (2001) sostiene que la educación ambiental puede ser una vía para la transformación de la sociedad y la cultura. La educación ambiental presenta la entereza de suponer que las problemáticas ambientales todavía pueden solucionarse y hasta prevenirse. Sin embargo, no sugiere de forma particular los mecanismos para lograrlo o los objetivos que deben trazarse para una humanidad armonizada con el planeta.

En el contexto de la educación ambiental, se presenta la problemática del planeta y se brindan algunas sugerencias prefabricadas para cambiar la realidad. Opciones como el reciclaje y reforestación son algunas premisas que se escuchan con frecuencia y que han sido tomadas como programas estatales intentando romper records internacionales (La Tribuna, 2016). A través de la autorreflexión es posible generar ese cambio. Pero se necesita ir un paso más adelante de la crítica generando una propuesta particular, de lo contrario, se corre el riesgo de continuar el círculo vicioso de "criticar la crítica". Esto puede ejemplificarse por medio de las campañas de reforestación, 
en las cuales muchas personas sembraron árboles (criticaron la realidad) pero no les dieron el seguimiento necesario a las plantas y estas murieron. Siendo así que la crítica no dio el siguiente paso hacia la responsabilidad colectiva y permanente.

Se hace necesario enlazar la crítica con un interés de mantenerse en el camino de cambiar y mejorar. Estas propuestas de cambio pueden relacionar la educación ambiental de manera directa con los Objetivos de Desarrollo del Milenio de las Naciones Unidas. Esta declaración recoge los aspectos relacionados con el desarrollo sostenible y la erradicación de la pobreza (Robles Llamazares, 2007). Al inferir que el desarrollo sostenible debe ser integral, es oportuno hablar de esperanza con base en los temas que son de interés para todas las personas.

Estos temas presentan puntos de partida para comunidades más justas y empoderadas en sus capacidades. De ahí que las Naciones Unidas los defina en ocho enunciados: 1) Erradicar la pobreza extrema y el hambre. 2) Lograr la enseñanza primaria universal. 3) Promover la igualdad entre los géneros y la autonomía de la mujer. 4) Reducir la mortalidad infantil. 5) Mejorar la salud materna. 6) Combatir el VIH/ SIDA, el paludismo y otras enfermedades.7) Garantizar la sostenibilidad del medio ambiente. 8) Fomentar una asociación mundial para el desarrollo.

Estos ocho objetivos se interrelacionan y abordan la temática de las necesidades esenciales y ayudan a elaborar los deseos de la población con respecto a su futuro. Si bien se dice que en Honduras "la gente vive el día a día" - premisa que tiene cierta validez - las personas en particular al ser abordadas comentan deseos particulares como una mejor casa, éxito escolar para sus hijos, salud, agua permanente y trabajo digno, entre otros. Todos estos deseos son cubiertos en los Objetivos de Desarrollo del Milenio y amparan la sombrilla del desarrollo sostenible. 


\section{El educador y la responsabilidad moral de la esperanza}

LeGrange (2011) comenta que los educadores se enfrentan ante tres responsabilidades morales: las poblaciones rurales, las dificultades arrastradas del siglo XX y el empoderamiento de los retos del siglo XXI. Estas responsabilidades se interpretan como la necesidad de acercar a las comunidades rurales al mundo contemporáneo e insertarlas en la sociedad logrando que conserven su cultura. Debe asistirse a estas poblaciones en la inserción que no se hizo posible en el siglo XX por la desesperanza e insensibilidad capitalista. Solo así se podrá vislumbrar un desarrollo sostenible inclusivo ante la realidad del siglo actual. Se debe ayudar a las personas a reconocer no solo su naturaleza oprimida sino su posición como sujetos tendentes a cambiar esa realidad (Freire, 1998). Al abordar esta necesidad con esperanza por medio de un enfrentamiento serio y crítico en donde los participantes son auténticos ante la sociedad se convierten en entidades con presencia y voz en un mundo injusto.

La esperanza debe centrarse como un complemento a la crítica si se desea escapar del cinismo que transmite el espectro político actual. La esperanza es necesaria para una pedagogía que vaya más allá de la injusticia, la desigualdad y la falta de oportunidad del pasado y el presente. Bloch (1986) pensaba que se debe ir más allá de la condenación del presente para ofrecer una visión alternativa del futuro en donde se capturen la imaginación y los deseos profundos de las personas. Por ello se hace necesario brindar una alternativa que visualice los fracasos del pasado y ofrezca un camino provisional de acción presente hacia la superación del fracaso en el futuro inmediato.

Se vive en un planeta que está cambiando, sí, se vive en un planeta donde la vida silvestre está perdiéndose, entonces ¿Qué se hará al respecto? Los educadores deben hacer más que advertir a sus educandos, deben darles la esperanza y la fuerza para trabajar en transformar el mundo. Freire (1998) pensaba que si el ser humano está condicionado, no está determinado, y por ende es libre para rebelarse contra ese condicionamiento. Freire aboga por una realidad 
fundada en el diálogo donde los individuos trabajen en colectividad y solidaridad, donde primero imaginen su realidad inmediata y trabajen en equipo por cambiarla. Esta construcción al enlazarse con los Objetivos de Desarrollo del Milenio incide en la realidad inmediata de todas las poblaciones. La esperanza es el medio para construir un movimiento progresivo que puede romper el ciclo del cinismo y duda, y así inspirar a las personas hacia una emancipación colectiva. Marcuse (1972) estima necesario convencer a las personas que la verdadera libertad no es una búsqueda individual, sino que un esfuerzo que abarca toda la comunidad en donde la realidad es la motivación para cambiar, y no la fuente de la represión.

\section{La Educación No Formal y la Esperanza}

La educación no formal es atendida por organizaciones cuyo propósito es el cambio social a través de acciones de alimentación, salud y educación. Conforman un abanico de posibilidades y prácticas que hace difícil generalizar características básicas a su alrededor. A nivel mundial, las ONG's llevan la responsabilidad de la educación ambiental no formal (Valentini y Martelli, 2016). Por lo tanto, los educadores no formales y los supervisores necesitan conocer la cultura de sus estudiantes puesto que es imposible realizar una educación no formal efectiva basada en conceptos importados o desconociendo la realidad inmediata de la comunidad.

Boggs (1991) plantea que la educación no formal tiene como propósito promover las actitudes y capacidades necesarias para participar y desenvolverse en una sociedad democrática, y que el educador debe dejar de ser pasivo e involucrarse de manera activa en el medio que se desenvuelve. El ambiente no formal es ideal para equilibrar la tendencia debido a la plasticidad de su temática y la libertad de metodologías de enseñanza. Quigley (2000) piensa que los educadores deben informar e imaginar una mejor sociedad y así construir un mejor futuro a través de un involucramiento activo en los sistemas democráticos. Al conocer la realidad inmediata de los educandos se construye con ellos un derrotero particular que llene sus expectativas, 
supere sus dificultades y genere prosperidad a través de los Objetivos de Desarrollo del Milenio.

Dave et al. (1990) sugieren que la educación no formal no existe en un vacío social y por lo tanto, para ser eficaz debe tener lazos horizontales fuertes con la comunidad como la familia, las personas mayores, las instituciones religiosas y políticas, los empleados, los sucesos sociales y culturales, los medios de comunicación y las características ambientales. En este sentido Pérez-Luna y Sánchez-Carreño (2005) sostienen que la educación no formal se basa en lo que ocurre en la vida, y por eso prepara al hombre para actuar en ella. La educación no formal tiene que impulsar la reflexión sobre el pensamiento, el ser, la realidad y el cambio, de manera que produzcan movimientos cualitativos del pensar, y por lo tanto, del actuar sobre la realidad. Sumado a lo anterior Brookfield (2000) considera que la reflexión crítica es una práctica cimentada en el proceso de autorreflexión. Esta reflexión debe ser un proyecto colaborativo porque en el fondo es un proceso social, sin embargo, se corre el peligro de sucumbir al pesimismo al ver que la situación es tan compleja y que requiere de tanto trabajo. De hecho, el cambio es complejo y sí requiere trabajo, pero una motivación que incida sobre los deseos de prosperidad de una población, tendrá mejor asidero y capacidad de éxito.

\section{La esperanza de un mundo para todos compartido}

González-Gaudiano (2013) sostiene que, si el problema está ocasionado por todos, de todos debe surgir la solución. La complejidad radica en determinar la responsabilidad y obligación de cada uno; en este sentido, un campesino no presenta la misma "huella de Carbono" que un citadino o este ante una empresa petroquímica. A partir de esto nace el principio de "responsabilidad compartida, pero diferenciada". Al vivir en una sociedad tan dividida muchas veces se escucha la expresión "yo pongo mi granito de arena" en el reciclaje, en el ahorro del agua, etc. 
En vista de ello, González- Gaudiano (2013) establece que esta manera de pensar socava la acción colectiva y retrasa la generación de ideas que lleven a un cambio. Las buenas prácticas aisladas y en silencio no tienden a resolver el problema sino que únicamente "limpian la conciencia del individuo y pintan de verde" los comportamientos consumistas. Nuevamente cabe el ejemplo de las reforestaciones masivas, en las cuales las personas asisten para tomarse la fotografía con un arbolito por la publicidad que genera en las redes sociales y de esa manera lavan su "yo pecador" de la culpa ambiental; tomada la foto termina el interés por el planeta. Toda la humanidad tiene una responsabilidad y obligación ante el planeta y es imperativo pagar esa deuda moral.

Bajo este concepto de conciencia aislada es que se convierte en una necesidad transformar a la educación ambiental en una plataforma total, la que no necesariamente se convierta en una nueva asignatura del currículo, sino que la sociedad tome conciencia y se empodere de la educación ambiental como parte de su realidad personal y nacional. Novo (1995) piensa que es imprescindible vincular los problemas ambientales con la economía internacional y sobre todo con los modelos de desarrollo. Siendo en el caso de la educación no formal, conseguir esa vinculación transversal desde su cimiento a través de los Objetivos de Desarrollo del Milenio.

Los problemas del planeta no deben verse solamente como referencia a sus consecuencias, sino analizar de dónde se originan, y se sabe que son originados en un capitalismo alimentado por la sociedad de consumo. En la medida en que las personas se den cuenta que se puede vivir con menos - y que esta idea se propague entre las masas - conseguirá así un verdadero cambio en la manera de pensar, siendo así que se logrará una visión holística y entrelazada del hombre con la naturaleza. Por tal motivo, Marcuse (1972) sugiere que es necesario regresar a la naturaleza y abogar por una relación simbiótica con ella, en donde se rompa la idea de la subordinación de la naturaleza ante el hombre, y con la idea del hombre dominando al hombre. Se hace necesario inspirar al educando hacia una apreciación de la naturaleza, 
insertándolo en ella para que observe la relación que esta tiene consigo mismo. De esta manera, el educando se vincula con la visión ecocéntrica que ofrece placer y satisfacción más allá de las comodidades materiales.

Debe entenderse que la capacidad de carga de los ecosistemas es equivalente a la capacidad de riqueza de una comunidad. Si bien los Objetivos de Desarrollo del Milenio apuntan hacia la satisfacción de las necesidades esenciales, se debe afrontar con una actitud crítica y realista el concepto del límite. Es decir, que no todas las necesidades pueden ser satisfechas pero sí se puede mejorar significativamente en otros ámbitos. Por tanto, necesidades y límites empiezan ya a jugar un papel importante en la interpretación de la problemática ambiental desde el ámbito educativo.

Hacia una educación ambiental crítica y esperanzadora

En el año 1992 se estableció que la educación ambiental es un acto político basado en valores para la transformación social por tanto es imposible continuar trabajando simplemente para dar información y crear opiniones (McKay, 2013). No basta crear opiniones, es crucial trabajar para la toma de decisiones. Esa es la dimensión política de la educación ambiental; lo que se construye con un pequeño colectivo en el salón de clases repercute sobre el barrio y la comunidad. Desde esta perspectiva, la siguiente fórmula "Armonía entre los seres humanos y la naturaleza" orienta la tarea educativa. En efecto, no es posible la solidaridad con la naturaleza si los seres humanos no se plantean la solidaridad entre ellos. Se sugiere por lo tanto que la función política y crítica de la educación ambiental puede funcionar a través de estos cinco preceptos:

El hombre que es parte del planeta. Comprender que lo que sucede en cualquier parte del mundo repercute en el resto del planeta, por ende las necesidades de la humanidad se insertan en un conjunto planetario en donde nadie es ajeno a los problemas. Así se comprenderá que el planeta es un sistema cerrado que, si bien recibe energía del exterior 
no intercambia materia. Todo lo que sucede en una parte repercute en la totalidad del sistema (los residuos arrojados, la contaminación, la pobreza, etc.).

Crecimiento y desarrollo como definiciones diferentes. Entender que el desarrollo no solo se liga a la adquisición de bienes materiales particulares sino al protagonismo social y calidad de vida en términos ambientales. Resaltar la idea de calidad de vida, abandonando la identificación con el nivel de vida medido a través de indicadores cuantitativos como automóvil lujoso, televisor plasma, teléfono móvil del año etc. Calidad de vida entendida desde la perspectiva de "ser más" y no desde la de "tener más". Calidad de vida orientada a la recuperación de los valores esenciales para la felicidad humana como la armonía y el equilibrio en el uso del entorno, en las relaciones con los otros, en el desempeño de las propias tareas, en la organización de los núcleos familiares y sociales, entre otros.

Sociedades justas, responsables con el planeta a través de la equidad. Lograr que las personas acepten su lugar dentro del planeta como seres integrados al ecosistema. Al entender la naturaleza, se aceptará al vecino. Entendiendo que para adoptar estos valores se requiere tener satisfechas las necesidades básicas esenciales como alimento, vivienda, educación, salud y trabajo. A través de los Objetivos de Desarrollo del Milenio se puede construir las ideas que lleven a la acción. Esto se podría lograr empoderando la idiosincrasia particular de cada comunidad para incorporarla en la toma de decisiones nacionales. El sistema mundial requiere una orientación hacia un nuevo paradigma interpretativo de las relaciones humanidad - naturaleza.

El valor de la diversidad. Reconocer la diversidad cultural como un elemento esencial de la biodiversidad y no solo en el plano biológico. Desde esta perspectiva, la pérdida de diversidad que están sufriendo los ecosistemas, con un alarmante ritmo de extinción de especies y el arrasamiento de muchas culturas, debería ser objeto de programas educativos - ambientales que ayudasen a las personas a comprender cómo esa diversidad tiene un valor intrínseco y se relaciona 
directamente con la estabilidad de los sistemas y sus posibilidades de mantenimiento en condiciones de equilibrio a lo largo de la historia.

La Educación como un derecho de todos los seres humanos. Implementar no sólo la educación en general, sino una educación informada y actual, que contribuya al esclarecimiento de la crisis desde la búsqueda de sus causas profundas de orden ético, económico y científico. Una educación que permita la identificación de los modelos de actuación que han creado y siguen creando en la actualidad las actitudes depredadoras de una parte de la humanidad sobre la otra y en su conjunto, sobre el resto del planeta.

Nieto-Caraveo (2003) considera que no se puede esperar movimientos sociales que se expresen conforme a una identidad uniforme, con principios y dogmas homogéneos. Las posiciones rígidas y cerradas solo dañan la posibilidad de construir consensos y acuerdos sociales, debido a que cada cultura y comunidad presenta problemas particulares que no pueden ser tratados de la misma manera. Conociendo los movimientos particulares se puede comprender mejor las luchas, las alternativas y las diferentes tensiones que en la sociedad generan los temas ambientales. Un educador no formal seguro fracasará en su objetivo de crear conciencia si desconoce la realidad inmediata de la comunidad o si piensa que una estrategia que tuvo éxito en un lugar, tendrá la misma tasa de resultado en otro. Los educadores deben estar conscientes que cada realidad es diferente, cada contexto es similar, y que la manera de pensar de las comunidades varía entre una y otra.

Se debe trabajar para que las personas tengan mayor información y opinión. Trabajar para que sean capaces de tomar decisiones sobre la información y sobre las opiniones que comparten. Para lograr insertar la esperanza se debe entender el problema. Por ende es necesario criticar el sistema y a partir de ahí forjar la esperanza de un mundo mejor. No se estima conveniente generar estrategias de educación ambiental a partir de manuales convencionales sin tener una idea clara de las necesidades de las comunidades ya que se corre el peligro de 
cerrarles la puerta ante el aprendizaje y acrecentar el pesimismo. De ahí que una aproximación bancaria tradicional hacia la enseñanza fracasará a pesar de que los educandos se aprecien atentos.

La educación ambiental es un proceso particular y específico para cada sector de la población. Un acercamiento propio para el occidente del país no será el apropiado para la costa caribeña debido a las diferencias culturales de ambas. Sin embargo, todas las poblaciones de Honduras se enfrentan ante las mismas disyuntivas críticas para el desarrollo humano y la conservación de los recursos naturales. Se sugiere que cualquier estrategia de educación ambiental no formal sea particular y basada en la realidad de las comunidades a concienciar. Un acercamiento previo a las poblaciones y conversaciones con sus líderes (patronatos, juntas de agua, maestros, clérigo, autoridades, etc.) construirá la base para el éxito ya que el educador podrá observar de primera mano las necesidades, anhelos, dificultades y potencial de las comunidades.

Se vuelve necesidad forjar una filosofía ambiental que recobre el sentido del hombre en la realidad y responda a una conceptualización integral de la calidad de vida acorde con las expectativas del futuro. Para crear esta filosofía ambiental, el educador en el campo debe construir con base en los Objetivos de Desarrollo del Milenio las herramientas de orientación ciudadana, y así generar de manera inicial los contenidos en los que estima sensibilizar. A partir de esto, permitir a los educandos que reflexionen y discutan de manera clara las necesidades y problemas de su comunidad con el fin de tomar medidas efectivas para implementar en ella los cambios pertinentes consensuados en los espacios críticos de reflexión y construcción.

\section{Conclusiones}

La educación ambiental es una fuerza por la ética planetaria la que si logra fusionarse con la esperanza se convierte en una estrategia sociopolítica que apunta hacia la consecución de las necesidades 
elementales de la humanidad. Estas necesidades pueden ilustrarse a través de los ocho Objetivos de Desarrollo del Milenio de las Naciones Unidas los cuales abordan temáticas transversales aplicables a todas las culturas. Para lograr esta activación, la educación ambiental, debe ser particular y estructurada a las necesidades específicas del educando, nunca suscrita a un protocolo o idea nacional.

La esperanza aplicada a la educación ambiental no formal puede ser una herramienta efectiva si se aplica dentro del contexto especial del cambio y a la idea por cambiar a partir de un escenario informado de la realidad. Forzada y estandarizada la educación ambiental pierde el objeto. La educación ambiental no puede ser efectiva si se orienta únicamente en la sistematización e implantación del temor, de la impregnación de un sentimiento de culpa por la situación actual del planeta y de la crítica hacia los errores del pasado. La educación ambiental debe informar, criticar, plantear, forjar y actuar sobre las circunstancias particulares en las que se desea ejercer una influencia positiva hacia el cambio de paradigma de humanidad y sociedad.

La educación no formal al ser ecléctica en su naturaleza y llevada a cabo por entidades que no están necesariamente sometidas a políticas estatales, se presta para abordar a un grupo más amplio de educandos y le facilita tener una mayor libertad de modelos de enseñanza. Puede abordar temáticas que cubran necesidades específicas que apunten hacia el cambio en la manera de pensar y actuar de los educandos. La educación no formal tiene el potencial de generar un cambio acelerado en las comunidades debido a que aborda a los diversos grupos socioculturales, y consulta directamente a los adultos quienes toman las decisiones a nivel de núcleo familiar.

La esperanza de un mundo mejor debe ser practicada de forma consistente y consciente, de manera que la esperanza se convierta en un vehículo hacia la plenitud. En este caso, el educador deber ser proactivo y ser la luz que brilla en un mar de oscuridad guiando al mundo a abandonar el letargo del pesimismo. El temor genera pesimismo y conformidad. La conformidad es una forma de auto 
opresión que evita que la persona logre a plenitud sus sueños al acomodarse en una realidad incompleta. La conformidad se destruye al incidir en la creación de una sociedad más justa y ecológicamente equilibrada en donde sus habitantes son conscientes de su propia voz y de su preponderancia en las decisiones de la sociedad.

Los modelos políticos acosan a la humanidad con necesidades que confunden la calidad de vida con medios de vida. Esta idea de "tener más" en vez de "ser más" es insostenible y aleja a la humanidad de la comprensión acerca de cómo las acciones depredadoras afectan al planeta y traen a la larga consecuencias. La educación ambiental debe plantear un mundo en el que se garanticen las necesidades elementales - y no necesariamente que todos tengan lo mismo - pues la igualdad no es lo mismo que la equidad, pero sí es pertinente que todos sean responsables con lo que tienen y con el uso que le dan a sus recursos. Ser bueno y responsable está bien, pero esa idea de ética ambiental debe multiplicarse e incidir de manera general en todos los estratos culturales y sociales.

Criticar las circunstancias, reflexionar sobre la actualidad, construir la esperanza y cambiar la realidad son las herramientas mentales que llevarán a un futuro sostenible. El mundo está en crisis, pero la vida en el planeta no se termina. La humanidad está en crisis y su tiempo se agota. La esperanza es el cimiento y antídoto de la negatividad. La vida en la tierra no va a terminarse con la salida del humano. El humano debe entender que no es fundamental para los ciclos terráqueos; siendo así que, si la vida en la tierra continúa, la humanidad podrá seguir usándola como hábitat solo sí el hombre despierta de su pesimismo.

A través de la esperanza en la educación ambiental no formal se busca eliminar sistemáticamente el temor y la incertidumbre, dejar atrás la culpa de los errores pasados, el pesimismo del conformismo y abrigar la esperanza de que lograr las necesidades elementales es posible. La desesperanza se reemplaza con positivismo y optimismo, el cual se transforma en ideas y satisfacción por el trabajo. La imaginación es una característica de los seres humanos. Es fundamental despertar 
esa capacidad natural para soñar. El educador es llamado a despertar la esperanza en cada persona que encuentre a su paso y a la vez mantener viva esa esperanza aun en las instancias más oscuras. La extinción pasará, el cambio climático pasará, pues son procesos que ya no pueden retrocederse. Lo hecho, hecho está. ¿Cómo va el humano a cambiarlo? En este sentido, el educador debe ser la chispa que enciende la luz interior de la humanidad para que esta encuentre su sendero.

La esperanza es lo único que no se pierde. Un mundo mejor es posible cuando el humano se dé cuenta de su lugar dentro del ecosistema. La voluntad de ser mejores es innata en cada uno. El educador ambiental y el educando forjan su destino construyéndolo de acuerdo a sus necesidades particulares y a las de la comunidad. Sus acciones son el efecto dominó que desencadena en la construcción del potencial del ser que en este momento necesita la humanidad para enfrentar las vicisitudes del futuro. 


\section{Referencias Bibliográficas}

Boggs, D.L. (1991). Adult Civic Education. Thomas Books, Springfield, IL.

Bloch, E. (1986).The Principles of Hope. Cambridge, MA: MIT Press.

Brookfield, S.D. (1998). Critical y Reflective Practice. Journal of Continuing Education in the Health Professions18 (4): pp.197-205

Dave, R., Ranaweera, A. y Sutton, P. (1990). Educación no formal. Chasqui, Revista Latinoamericana de Educación. (36): pp.35-40

Fernández Bregón, N. 2011. Eficiencia energética, presente y futuro: Propuesta de educación ambiental para un ámbito no formal. Trabajo de fin de Máster (UAL), Máster Interuniversitario Andaluz en Educador/ a Ambiental: p.72

Freire, P. (1996). Pedagogía de la esperanza, un reencuentro con la pedagogía del oprimido. Siglo XXI Editores, México. p.150

Freire, P. (1998). Politics and Education. Los Ángeles: UCLA Latin American Center Publications.

La Prensa. (10 de junio 2017). Honduras, entre los países más afectados por el calentamiento. La Prensa edición digital. Recuperado de http:// www.laprensa.hn/honduras/1079209-410/honduras-calentamientocambio_climatico-inseguridad_alimentaria-acuerdo_par\%C3\%ADs

La Tribuna. (31 de mayo 2016). Masiva campaña para reforestar "Honduras siembre vida. La Tribuna edición digital. Recuperado de http:// www.latribuna.hn/2016/05/31/masiva-campana-reforestar-hondurassiembre-vida/ 
LeGrange, L. (2011). Pedagogy of hope after Paulo Freire. South African Journal of Higher Education (25)1:pp.183-189.

Marcuse, H. (1972). Counter revolution and Revolt. Boston: Beacon Press. pp. 139

McKay, J. (2013). Learning for survival, resilience, well-being and continuance: An epistemology and pedagogy for environmental education/education for sustainability informed by Mâori Culture. Trabajo de tesis para optar al título de Doctor en Filosofía de la Universidad de Otago, Nueva Zelanda.

Nieto-Caraveo, L. (2001). Presentación, en: dos Santos, J., Sato, E.y Sato, M. Am contribucao de educacao ambiental a espernanca de Pandora. Rima Editora.

Nieto-Caraveo, L. (2003). Ambientalismo(s) ¿Ser o no ser? Pulso, Diario de San Luis Potosí, México. Recuperado de http://ambiental.uaslp.mx/ docs/LMNC-AP030925.pdf

Novo, M. (1995). La educación ambiental. Bases éticas, conceptuales y metodológicas. Madrid, España: Editorial Universitas.

Pain, R. (2009). Globalized fear? Towards an emotional geopolitics. Progres in Human Geography. 33(4): pp.466-486

Pérez-Luna, E. y Sánchez-Carreño, J. (2005). La educación comunitaria: una concepción desde la Pedagogía de la Esperanza de Paulo Freire. Revista Venezolana de Ciencias Sociales 9(2):pp. 317-329

Reyes García, J. (1995). En torno a Paulo Freire: Aspectos filosóficos de su pensamiento y aportes a la reflexión de raíz latinoamericana. Tesis de Pregrado para Licenciatura en Filosofía. Pontificia Universidad Católica de Chile. 
La Educación Ambiental no Formal orientada en la Pedagogía de la Esperanza

Robles Llamazares, M. (2007). Objetivos de desarrollo del milenio. Humanismo y Trabajo Social, 5: pp. 93-101.

Valentini, V. y Martelli, J. (2016). The contribution of NGOS in environmental education: an evaluation of stakeholders' perception. Ambiente. Soc 19(3): pp. 47-66

VanHeertum, R. (2006). Marcuse, Bloch and Freire: reinvigorating pedagogy of hope. Policy Futures in Education, 4(1):pp. 45-50.

<Paradigma> - Revista de Investigación Educativa. Año 24. No. 38 